\section{Correspondence on 'Risk of systemic lupus erythematosus after immune thrombocytopenia and autoimmune haemolytic anaemia: a nationwide French study'}

We read with great interest the articles by Zhu et al ${ }^{1}$ and Mo et $a l^{2}$ studying the risk of new systemic lupus erythematosus (SLE) diagnosis after immune thrombocytopenia (ITP) and autoimmune haemolytic anaemia (AIHA) diagnoses in the Taiwanese National Health Database. Zhu et al observed 34 SLE cases among 723 patients with incident ITP between 2000 and 2013. The 1-year and 5-year cumulative incidences were $1.9 \%$ and 4.8\%, respectively. ${ }^{1}$ Mo et al reported the diagnosis of 117 SLE during the follow-up of 731 patients with incident AIHA between 2005 and 2012. The 1-year and 5-year cumulative incidences were $11.3 \%$ and $17.3 \%$, respectively. ${ }^{2}$

In the present study, we estimated the risk of new SLE after incident ITP or AIHA in the entire French general population. FAITH and AHEAD cohorts ${ }^{3}$ are the French nationwide cohorts of adult patients with incident primary ITP and AIHA, respectively. These cohorts are built using validated algorithms in the French health database (Système National des Données de Santé), which links sociodemographic, outhospital and in-hospital data for the entire French population (>66 million inhabitants). ${ }^{4}$ SLE was defined by the date of first in-hospital or chronic disease (the latter being encoded by general practitioners) M32 code of the International Classification of Diseases, V.10 (ICD-10). Patients have been included between 2009 and 2017 in FAITH and between 2012 and 2017 in AHEAD. Follow-up ended in December 2018 for all patients. The cumulative incidence of SLE after diagnosis of incident primary ITP/AIHA was computed by subgroups of age and sex using the cumulative incidence risk function taking into account the competing risk of death. ${ }^{5}$

Among 9589 adult patients with incident primary ITP, $55.1 \%$ were women and median age was 62 years (IQR: 39-77 years). We observed 172 new diagnoses of SLE during the 39224 patient-year follow-up. One-year and 5-year cumulative incidences were $1.03 \%$ (95\% CI: $0.84 \%$ to $1.25 \%)$ and $1.89 \%$ (95\% CI: $1.61 \%$ to $2.20 \%$ ), respectively (table 1 ). The highest cumulative incidence of SLE was observed in women aged 18-45 years (1-year and 5-year cumulative incidences: $2.79 \%$ (95\% CI: $2.12 \%$ to $3.60 \%$ ) and $5.30 \%$ (95\% CI: $4.28 \%$ to $6.46 \%$ ), respectively). Among the 4609 adult patients with incident primary AIHA, 57.6\% were women and median age was 72 years (IQR: 56-82 years). We observed 38 new diagnoses of SLE during the 13233 patientyear follow-up. One-year and 5 -year cumulative incidences were $0.48 \%$ (95\% CI: $0.31 \%$ to $0.71 \%$ ) and $0.99 \%$ (95\% CI: $0.69 \%$ to $1.37 \%$ ), respectively (table 1 ). The highest cumulative incidence of SLE was observed in women aged 18-45 years (1-year and 5-year cumulative incidences: 1.67\% (95\% CI: $0.75 \%$ to $3.28 \%)$ and $4.46 \%$ (95\% CI: $2.53 \%$ to $7.19 \%)$, respectively).

Our study showed a low risk of SLE in patients diagnosed with primary ITP/AIHA $(<2 \%)$, except in women of childbearing age (approximately 4.5\%). These estimates are much lower than those measured in the Taiwanese population. Ethnical specificities may partly explain these discrepancies. Indeed, the annual incidence of SLE in Taiwan is high, estimated at 8.1 per 100000 person-years between 2000 and 2007 using the Taiwanese Health Database. ${ }^{6}$ SLE accounted for $21 \%$ of secondary ITPs in adult patients at ITP diagnosis in Taiwan ${ }^{7}$ compared with $9 \%$ in France. ${ }^{3}$ Conversely, the number of patients with ITP and AIHA in the Taiwanese study were unexpectedly low compared with our study in France.

The cumulative incidence of SLE after primary ITP/AIHA also depends on age and sex. In our study, patients were older and there was a higher men:women ratio than in the Taiwanese studies, while SLE occurred mainly in women aged 18-45 years. Of note, the cumulative incidence of SLE in patients aged 18-45 years after primary AIHA was similar in women and men.

The main limitations of our study are the possibility of misclassifications, like in other studies using similar data sources. However, the identification of patients with ITP/AIHA in the SNDS has been validated with very good positive predictive values. $^{89}$

In conclusion, the cumulative incidence of SLE after the diagnosis of primary ITP/AIHA was low in our study. It mainly affects women of childbearing age after ITP diagnosis and both men and women between 18 and 45 years of age after AIHA diagnosis.

Table 1 Cumulative incidence of systemic lupus erythematosus after incident primary immune thrombocytopenia and autoimmune haemolytic anaemia in France

\begin{tabular}{|c|c|c|c|c|c|c|}
\hline \multirow[b]{2}{*}{ Patients } & \multicolumn{3}{|c|}{ Primary ITP } & \multicolumn{3}{|c|}{ Primary AIHA } \\
\hline & SLE/n & $\begin{array}{l}\text { 1-year risk, } \\
\%(95 \% \mathrm{Cl})\end{array}$ & $\begin{array}{l}\text { 5-year risk, } \\
\%(95 \% \mathrm{Cl})\end{array}$ & SLE/n & $\begin{array}{l}\text { 1-year risk, } \\
\%(95 \% \mathrm{Cl})\end{array}$ & $\begin{array}{l}\text { 5-year risk, } \\
\%(95 \% \mathrm{Cl})\end{array}$ \\
\hline Women & $150 / 5285$ & 1.6 (1.3 to 2.0$)$ & 2.9 (2.5 to 3.5$)$ & $28 / 2653$ & $0.6(0.3$ to 0.9$)$ & 1.2 (0.8 to 1.7$)$ \\
\hline Men & $22 / 4304$ & $0.3(0.2$ to 0.5$)$ & $0.6(0.4$ to 0.9$)$ & $10 / 1956$ & $0.4(0.2$ to 0.7$)$ & 0.7 (0.3 to 1.3$)$ \\
\hline $18-45$ years & $102 / 2844$ & 2.0 (1.6 to 2.6$)$ & 3.8 (3.1 to 4.6$)$ & $23 / 699$ & 1.9 (1.0 to 3.1$)$ & 4.2 (2.7 to 6.3 ) \\
\hline $45-65$ years & $40 / 2374$ & 0.9 (0.6 to 1.4$)$ & 1.8 (1.3 to 2.4$)$ & 9/1017 & $0.5(0.2$ to 1.1$)$ & $0.9(0.4$ to 1.7$)$ \\
\hline Women & $31 / 1289$ & 1.3 (0.8 to 2.1$)$ & 2.4 (1.7 to 3.4$)$ & $8 / 589$ & 0.8 (0.3 to 1.9$)$ & 1.2 (0.6 to 2.4$)$ \\
\hline Men & $9 / 1085$ & $0.5(0.2$ to 1.0$)$ & $1.0(0.5$ to 1.9$)$ & $1 / 428$ & 0 & 0.4 (0.0 to 2.3 ) \\
\hline$\geq 65$ years & $30 / 4371$ & $0.4(0.3$ to 0.6$)$ & 0.7 (0.5 to 1.0$)$ & $6 / 2893$ & 0.1 (0.0 to 0.3$)$ & 0.2 (0.1 to 0.5$)$ \\
\hline Women & $23 / 2086$ & 0.7 (0.4 to 1.1$)$ & 1.1 (0.7 to 1.7$)$ & $5 / 1646$ & 0.2 (0.1 to 0.5$)$ & 0.3 (0.1 to 0.7$)$ \\
\hline
\end{tabular}

AlHA, autoimmune haemolytic anaemia; ITP, immune thrombocytopenia; SLE, Systemic lupus erythematosus. 
Julien Maquet $\odot,^{1,2}$ Margaux Lafaurie $\odot,^{2,3,4}$ Laurent Sailler, ${ }^{1,2,4}$ Maryse Lapeyre-Mestre $\odot^{2,3,4}$ Guillaume Moulis ${ }^{1,2,4}$

${ }^{1}$ Internal Medicine, University Hospital Centre Toulouse, Toulouse, Occitanie, France ${ }^{2} \mathrm{CIC}$ 1436, University Hospital Centre Toulouse, Toulouse, Occitanie, France ${ }^{3}$ Clinical Pharmacology, University Hospital Centre Toulouse, Toulouse, Occitanie, France

${ }^{4}$ UMR 1027, INSERM - University of Toulouse, Toulouse, France

Correspondence to Julien Maquet, Internal Medicine, University Hospital Centre Toulouse, Toulouse 31300, France; maquet.j@chu-toulouse.fr

Acknowledgements The authors thank Marjorie Boussac and the Caisse Nationale de l'Assurance maladie des Travailleurs Salariés engineers who performed raw data extraction from the SNDS database.

Contributors JM, ML and GM designed the study, carried out the data management and statistical analyses and wrote the paper. LS wrote the protocol of the FAITH cohort. GM wrote the protocol of the AHEAD cohort. All authors participated in the interpretation of the results, critically reviewed the manuscript and gave final approval for submission.

Funding This study is academic, funded by Société nationale française de médecine interne (SNFMI) and the Toulouse University Hospital.

Competing interests This study is academic. JM reports grants from SNFMI. GM received meeting attendance grants from Novartis and Amgen and is coordinator of research studies granted by CSL Behring, Novartis and Grifols. He participated in educational sessions funded by Amgen and Novartis, and boards for Novartis and Amgen.

Patient and public involvement Patients and/or the public were not involved in the design, or conduct, or reporting, or dissemination plans of this research.

Patient consent for publication Not required.

Ethics approval According to French law, authorisations were obtained in 2012 for the FAITH cohort from the Institut des Données de Santé (no 40) and the Commission Nationale de l'Informatique et des Libertés (DE-2012-076) and in 2019 for the AHEAD cohort from the Comité d'Expertise pour les Recherches, les Etudes et les Evaluations dans le domaine de la Santé and the Commission Nationale de I'Informatique et des Libertés (DR-2019-229).

Provenance and peer review Not commissioned; internally peer reviewed. (c) Author(s) (or their employer(s)) 2020. No commercial re-use. See rights and permissions. Published by BMJ.

A Check for updates
To cite Maquet J, Lafaurie M, Sailler L, et al. Ann Rheum Dis Epub ahead of print: [please include Day Month Year]. doi:10.1136/annrheumdis-2020-219470

Received 8 November 2020

Accepted 19 November 2020

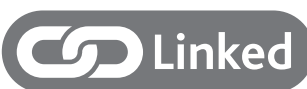

http://dx.doi.org/10.1136/annrheumdis-2020-219583

Ann Rheum Dis 2020:0:1-2. doi:10.1136/annrheumdis-2020-219470

\section{ORCID iDs}

Julien Maquet http://orcid.org/0000-0002-2187-9435

Margaux Lafaurie http://orcid.org/0000-0001-6010-2891

Maryse Lapeyre-Mestre http://orcid.org/0000-0002-5494-5873

\section{REFERENCES}

1 Zhu F-X, Huang J-Y, Ye Z, et al. Risk of systemic lupus erythematosus in patients with idiopathic thrombocytopenic purpura: a population-based cohort study. Ann Rheum Dis 2020;79:793-9.

2 Mo H-Y, Wei JCC, Chen X-H, et al. Increased risk of systemic lupus erythematosus in patients with autoimmune haemolytic anaemia: a nationwide population-based cohort study. Ann Rheum Dis 2020. doi:10.1136/annrheumdis-2020-218886. [Epub ahead of print: 22 Sep 2020].

3 Moulis G, Palmaro A, Montastruc J-L, et al. Epidemiology of incident immune thrombocytopenia: a nationwide population-based study in France. Blood 2014:124:3308-15.

4 Bezin J, Duong M, Lassalle R, et al. The National healthcare system claims databases in France, SNIIRAM and EGB: powerful tools for pharmacoepidemiology. Pharmacoepidemiol Drug Saf 2017;26:954-62.

5 Coviello V, Boggess M. Cumulative incidence estimation in the presence of competing risks. Stata J 2004;4:103-12.

6 Chiu Y-M, Lai C-H. Nationwide population-based epidemiologic study of systemic lupus erythematosus in Taiwan. Lupus 2010;19:1250-5.

7 Hung G-Y, Lee C-Y, Yen H-J, et al. Incidence of immune thrombocytopenia in Taiwan: a nationwide population-based study. Transfusion 2018;58:2712-9.

8 Mezaache S, Derumeaux $\mathrm{H}$, Ferraro $\mathrm{P}$, et al. Validation of an algorithm identifying incident primary immune thrombocytopenia in the French National health insurance database. Eur J Haematol 2017;99:344-9.

9 Maquet J, Derumeaux H, Lapeyre-Mestre M, et al. Validation of hemolytic anemia discharge diagnosis codes in the French Hospital database. Eur J Intern Med 2020;79:136-8 